\title{
A Stochastic Weakly Damped, Forced KdV-BO Equation
}

\author{
Guolian Wang \\ Department of Mathematics, Tongji University, Shanghai 200092, China \\ Correspondence should be addressed to Guolian Wang; guolianwang@126.com \\ Received 14 January 2014; Revised 13 April 2014; Accepted 13 April 2014; Published 5 May 2014 \\ Academic Editor: Tonghua Zhang \\ Copyright (C) 2014 Guolian Wang. This is an open access article distributed under the Creative Commons Attribution License, which \\ permits unrestricted use, distribution, and reproduction in any medium, provided the original work is properly cited. \\ We investigate the long time behavior of the damped, forced $\mathrm{KdV}-\mathrm{BO}$ equation driven by white noise. We first show that the global \\ solution generates a random dynamical system. By energy type estimates and dispersive properties, we then prove that this system \\ possesses a weak random attractor in the space $H^{1}(\mathbb{R})$.
}

\section{Introduction}

The deterministic Korteweg-de Vries-Benjamin-Ono (KdVBO) equation describes a large class of internal waves in the ocean and stratified fluid. The well-posedness for this equation was studied in $[1,2]$. When the surface of the fluid is submitted to a nonconstant pressure, or when the bottom of the layer is not flat, a forcing term has to be added to the equation $[3,4]$. The long time behavior of the forced generalized KdV-BO was studied in [5]. In this paper we are interested in the case when the forcing term is random. The well-posedness for the stochastic KdV-BO driven by the additive noise was studied in [6]. We have found no studies on the long time behavior of the stochastic KdV-BO equation.

In this paper, we consider the long time behavior of the following stochastic damped, forced $\mathrm{KdV}$-BO equation:

$$
\partial_{t} u+\alpha \mathscr{H}\left(\partial_{x}^{2} u\right)+\beta \partial_{x}^{3} u+u \partial_{x} u+\lambda u=f+\Phi \frac{\partial^{2} B}{\partial t \partial x},
$$

where $\alpha, \beta$, and $\lambda$ are real constants and $\alpha \beta \neq 0$. $\mathscr{H}$ denotes the Hilbert transform

$$
\mathscr{H} f(x)=p \cdot v \cdot \frac{1}{\pi} \int \frac{f(x-y)}{y} d y .
$$

The forcing term $f$ is time independent, and $u$ is a random process defined on $(t, x) \in \mathbb{R}^{+} \times \mathbb{R}$. $\Phi$ is a linear operator. Also, $B$ is a two-parameter Brownian motion on $\mathbb{R}^{+} \times \mathbb{R}$, that is, a zero mean Gaussian process whose correlation function is given by

$$
\mathbb{E}(B(t, x) B(t, x))=(t \wedge s)(x \wedge y),
$$

for $t, s \geqslant 0, x, y \in \mathbb{R}$.

Alternatively we consider a cylindrical Wiener process $W$ by setting

$$
W(t)=\frac{\partial B}{\partial x}=\sum_{i=0}^{\infty} \beta_{i} e_{i}
$$

where $\left\{e_{i}\right\}_{i \in \mathbb{N}}$ is an orthonormal basis of $L^{2}(\mathbb{R})$ and $\left\{\beta_{i}\right\}_{i \in \mathbb{N}}$ is a sequence of mutually independent real Brownian motions in a fixed probability space $(\Omega, \mathscr{F}, P)$ adapted to a filtration $\{\mathscr{F}\}_{t \geqslant 0}$.

Let us write the Itô form of (1) as follows:

$$
d u+\left(\alpha H\left(\partial_{x}^{2} u\right)+\beta \partial_{x}^{3} u+u \partial_{x} u+\lambda u-f\right) d t=\Phi d W
$$

with initial condition

$$
u(0, x)=u_{0}(x) .
$$

The purpose of this paper is to study the long time behavior of the problem (5) with initial data. Before describing our works, we recall some facts related to this paper. The Cauchy problem for the deterministic KdV-BO equation, that is, $\lambda=$ $f=\Phi=0$ in (5), was considered in [1]. There the authors obtained well-posedness results by using Fourier restriction norm method in Bourgain's type spaces $X_{s, b}$ with $b>1 / 2$. 
Based on the global existence results given in [1], the attractor of the damped forced KdV-BO equation was obtained in [5]. The stochastic KdV-BO equation (i.e., $\lambda=f=0$ in (5)) was studied in space $X_{s, b}$ with $b<1 / 2$ in [6]. By introducing some useful inequalities to deal with the irregularity caused by stochastic term, well-posedness results were obtained in [6]. Following the work in [6], we will construct the attractor to the Cauchy problem for the stochastic damped, forced $\mathrm{KdV}-\mathrm{BO}$ equation.

Before stating our main result precisely, we introduce some notations.

Denote by $(\cdot, \cdot)$ and $|\cdot|$ the inner product and the norm in $L^{2}(\mathbb{R})$, respectively. And $\|\cdot\|_{X}$ is the norm of the Banach space $X$.

Given two separable Hilbert spaces $\bar{H}$ and $\widetilde{H}$, we denote by $L_{2}^{0}(\bar{H} ; \widetilde{H})$ the space of Hilbert-Schmidt operators from $\bar{H}$ into $\widetilde{H}$. Its norm is given by

$$
\|\Phi\|_{L_{2}^{0}(\bar{H} ; \widetilde{H})}^{2}=\sum_{i \in \mathbb{N}}\left\|\Phi e_{i}\right\|_{\widetilde{H}}^{2}, \quad \Phi \in L_{2}^{0}(\bar{H} ; \widetilde{H}),
$$

where $\left(e_{i}\right)_{i \in \mathbb{N}}$ is any orthonormal basis of $\bar{H}$. When $\bar{H}=$ $L^{2}(\mathbb{R}), \widetilde{H}=H^{s}, L_{2}^{0}\left(L^{2}(\mathbb{R}) ; H^{s}\right)$ is simply denoted by $L_{2}^{0, s}$.

The proof of the global well-posedness of the solution (5)(6) is similar to [6]. Here, we only give the following global existence results.

Assume that $\Phi \in L_{2}^{0,0}$ (or $\left.\Phi \in L_{2}^{0,1}\right)$; let $u_{0} \in L^{2}\left(\Omega, L^{2}(\mathbb{R})\right.$ ) (or $u_{0} \in L^{2}\left(\Omega, H^{1}(\mathbb{R})\right)$ ) be $\mathscr{F}_{0}$-measurable. Then, the solution $u(t)$ is global and belongs to $L^{2}\left(\Omega ; C\left([0, T] ; L^{2}(\mathbb{R})\right)\right)$ (or $L^{2}\left(\Omega ; C\left([0, T] ; H^{1}(\mathbb{R})\right)\right)$ ) for any $T>0$.

We now give our main result about the long time behavior of the KdV-BO equation based on its global existence results.

Theorem 1. Under the assumption that $\lambda>0, f \in H^{1}(\mathbb{R})$, $\Phi \in L_{2}^{0,2}$, and $u_{s} \in L^{2}\left(\Omega ; H^{1}(\mathbb{R})\right)$ is $\mathscr{F}_{s}$-measurable, then the random dynamical system associated with the stochastic equation (5) with initial value $u(s)=u_{s}$ possesses a universal weak random attractor $\mathscr{A}$ in $H^{1}(\mathbb{R})$.

The paper is organized as follows. Section 2 contains some concepts about the random dynamical system, and Lemma 2 which gives the existence conditions and the structure of the attractor. Then we show that the unique solution of problem (5)-(6) generates a random dynamical system in Section 3. In Section 4, we prove that there exists a compact random absorbing set, which leads to the existence of a random attractor, that is, Theorem 1.

\section{Preliminaries on Random Dynamical System}

We now recall some concepts and results from [7-9].

Let $(\Omega, \mathscr{F}, P)$ be a probability space and $\left\{\theta_{t}: \Omega \rightarrow\right.$ $\Omega, t \in \mathbb{R}\}$ a family of measure preserving transformations such that $(t, \omega) \mapsto \theta_{t} \omega$ is measurable, $\theta_{0}=i d$, and $\theta_{t+s}=$ $\theta_{t} \circ \theta_{s}$ for all $s, t \in \mathbb{R}$. The flow $\theta_{t}$ together with the corresponding probability space $(\Omega, \mathscr{F}, P)$ is called a metric dynamical system.
A continuous random dynamical system (RDS) on a polish space $(X, d)$ with Borel sigma-algebra $\mathscr{F}$ over $\theta_{t}$ on $(\Omega, \mathscr{F}, P)$ is by definition a measurable map

$$
\Psi: \mathbb{R}^{+} \times \Omega \times X \longrightarrow X, \quad(t, \omega, x) \longmapsto \Psi(t, \omega) x,
$$

such that $P$-a.s.

(i) $\Psi(0, \omega)=i d$ on $X$;

(ii) $\Psi(t+s, \omega)=\Psi\left(t, \theta_{s} \omega\right) \Psi(s, \omega)$ for all $s, t \in \mathbb{R}^{+}$(cocycle property);

(iii) $\Psi(t, \omega): X \rightarrow X$ is continuous.

A set-valued map $K: \Omega \rightarrow 2^{X}$, the set of all subsets of $X$, is called a random compact set, if $K(\omega)$ is a compact $P$-a.s. and if $\omega \mapsto d(x, K(\omega))$ is measurable with respect to $\mathscr{F}$ for each $x \in X$, where $d(x, M)=\inf _{x \in M} d(x, y)$.

Let $\mathscr{A}(\omega)$ be a random set and $B \subset X$; one says $\mathscr{A}(\omega)$ attracts $B$ if

$$
\lim _{t \rightarrow \infty} \operatorname{dist}\left(\Psi\left(t, \theta_{-t} \omega\right) B, \mathscr{A}\right)=0, \quad P \text {-a.s. }
$$

A random set $\mathscr{A}(\omega)$ is said to be a random attractor for the RDS $\Psi$ if $P$-a.s.

(i) $\mathscr{A}(\omega)$ is a random compact set;

(ii) $\mathscr{A}(\omega)$ is invariant, that is, $\Psi(t, \omega) \mathscr{A}(\omega)=\mathscr{A}\left(\theta_{t} \omega\right)$, for all $t>0$

(iii) $\mathscr{A}(\omega)$ attracts all deterministic bounded sets $B \subset X$.

Similar to the deterministic theory, the existence result of random attractors can be stated as follows (see $[8,9])$.

Lemma 2. If there exists a random compact set absorbing every bounded set $B \subset X$, then the RDS $\Psi$ possesses a random attractor $\mathscr{A}(\omega)$,

$$
\mathscr{A}(\omega)=\overline{\mathrm{U}_{B \subset X} \Lambda_{B}(\omega)}
$$

where $\Lambda_{B}(\omega):=\cap_{s \geqslant 0} \overline{\cup_{t \geqslant s} \Psi\left(t, \theta_{-t} \omega\right) B}$ is the omega-limit set of $B$.

\section{Solve the Equations and Generate an RDS}

We consider the following linear problem to (5)-(6):

$$
(P)\left\{\begin{array}{c}
d \bar{u}+\left\{\alpha \mathscr{H} \partial_{x}^{2} \bar{u}+\beta \partial_{x}^{3} \bar{u} d t+\lambda \bar{u}\right\} d t=\Phi d W \\
\bar{u}(0)=0
\end{array}\right.
$$

whose solution is given by the stochastic integral (see [10])

$$
\bar{u}(t)=\int_{0}^{t} U(t-s) \Phi d W(s) .
$$

From now on we turn our attention to study the wellposedness of a weakly damped, forced $\mathrm{KdV}$-BO equation with random parameter by change of variable.

Let us study (5) by means of the change of variable

$$
v(t)=u(t)-\bar{u}(t)
$$


and then $u$ satisfies (5) if and only if $v$ is a solution of

$$
\begin{gathered}
v_{t}+\alpha \mathscr{H} v_{x x}+\beta v_{x x x}+\lambda v+v v_{x} \\
+\overline{u u}_{x}+v \bar{u}_{x}+\bar{u} v_{x}=f .
\end{gathered}
$$

This is a weakly damped, forced $\mathrm{KdV}-\mathrm{BO}$ equation with random parameter with the following initial condition:

$$
v(0, x)=u(0, x)+\bar{u}(0, x)=u(0, x) .
$$

The estimation about the random parameter $\bar{u}$ and the bilinear term $v v_{x}$ in (14) can be obtained by using the method in [6]. Then by the fixed point argument the global existence results to the random parameter Cauchy problem (14)-(15) can be obtained.

Theorem 3. Assume that $\Phi \in L_{2}^{0,0}$ (or $\left.\Phi \in L_{2}^{0,2}\right)$; let $v_{0} \in$ $L^{2}(\mathbb{R})$ (or $v_{0} \in H^{1}(\mathbb{R})$ ). Then, the solution $v(t)$ of problem (14) and (15) is global and belongs to $C\left([0, T] ; L^{2}(\mathbb{R})\right)$ (or $C\left([0, T] ; H^{1}(\mathbb{R})\right)$ for any $T>0$.

We summarize the above existence results for $P$-a.s. $\omega \in$ $\Omega$ of (14) with initial condition $v(s, x)=u(s, x)=v_{s}, s \in \mathbb{R}$, as follows.

(i) Under the assumption of Theorem 3, for $s<T$, and any $T \in \mathbb{R}$ and $v_{s} \in L^{2}(\mathbb{R})$, there exists a unique solution $v \in C\left([s, T] ; L^{2}(\mathbb{R})\right)$.

(ii) Under the assumption of Theorem 3, for $s<T$, and any $T \in \mathbb{R}$, and $v_{s} \in H^{1}(\mathbb{R})$, there exists a unique solution $v \in C\left([s, T] ; H^{1}(\mathbb{R})\right)$.

(iii) Denoting such a solution $v\left(t, \omega ; s, v_{s}\right)$, the mapping $v_{s} \mapsto v\left(t, \omega ; s, v_{s}\right)$ is continuous for all $s \leqslant T$.

Now we construct an RDS modeling the stochastic weakly damped, forced $\mathrm{KdV}-\mathrm{BO}$ equation. For example, consider a set of continuous functions with value 0 at $t=0$

$$
\Omega=\{\omega \in C(\mathbb{R}, \mathbb{R}): \omega(0)=0\} .
$$

Let $\mathscr{F}$ be the Borel sigma-algebra induced by the compact open topology of $\Omega$, and let $P$ be a Wiener measure on $(\Omega, \mathscr{F})$. We write $\left(\beta_{1}(t, \omega), \ldots, \beta_{k}(t, \omega), \ldots\right)=\omega(t)$. The time shift is simply defined by

$$
\theta_{s} \omega(t)=\omega(t+s)-\omega(s), \quad t, s \in \mathbb{R},
$$

and then $\left(\Omega, \mathscr{F}, P, \theta_{t}\right)$ is an ergodic metric dynamical system which models white noise.

Having the mapping $v_{s} \mapsto v\left(t, \omega ; s, v_{s}\right)$, we define

$$
u\left(t, \omega ; s, u_{s}\right)=S(t, s ; \omega) u_{s}=v\left(t, \omega ; s ; v_{s}\right)+\bar{u}(t, \omega),
$$

where $v\left(t, \omega ; s ; v_{s}\right)$ is a solution to (14) with $v(s)=v_{s}$ and $\bar{u}(t)$ satisfies

$$
\begin{gathered}
d \bar{u}+\left\{\alpha \mathscr{H} \partial_{x}^{2} \bar{u}+\beta \partial_{x}^{3} \bar{u}+\lambda \bar{u}\right\} d t=\Phi d W \\
\bar{u}(s)=0 .
\end{gathered}
$$

Obviously, for $s \leqslant r \leqslant t$, we have

$$
S(t, s ; \omega)=S(t, r ; \omega) S(r, s ; \omega) .
$$

Thanks to (17), for any $s, t \in \mathbb{R}^{+}, u_{s} \in H^{1}(\mathbb{R})$, we have $P$-a.s.

$$
S(t+s, 0 ; \omega) u_{0}=S\left(t, 0 ; \theta_{s} \omega\right) S(s, 0 ; \omega) u_{0} .
$$

Therefore, the process $\Psi: R^{+} \times \Omega \times V \rightarrow V$ defined by

$$
\Psi(t, \omega) u_{0}=S(t, 0: \omega) u_{0}
$$

is cocycle. It is continuous $\mathrm{RDS}$ on $H^{1}(\mathbb{R})$ over $(\Omega, \mathscr{F}, P$, $\left.\left(\theta_{t}\right)_{t \in \mathbb{R}}\right)$ and models the dynamical system associated with the stochastic equation (5) with initial value $u(s)=u_{s}$.

\section{Compact Random Absorbing Set}

In the following computations $\omega \in \Omega$ is fixed. We usually denote $L^{p}(\mathbb{R})(1 \leqslant p \leqslant \infty)$ by $L_{x}^{p} ; L^{p}\left([0, T] ; L^{q}(\mathbb{R})\right)(1 \leqslant$ $p \leqslant \infty, 1 \leqslant q \leqslant \infty)$ by $L_{t}^{p}\left(L_{x}^{q}\right) ;$ and $L^{p}\left(\mathbb{R} ; L^{q}([0, T])\right)(1 \leqslant$ $p \leqslant \infty, 1 \leqslant q \leqslant \infty)$ by $L_{x}^{p}\left(L_{t}^{q}\right)$ for any $T>0$ in this part.

First note that, for the Hilbert transform, we have for any $f, g \in L^{2}(\mathbb{R})$

$$
\begin{gathered}
\mathscr{H}^{2} f=-f, \quad \mathscr{H}(f g)=\mathscr{H}(\mathscr{H} f \mathscr{H} g)+f \mathscr{H} g+g \mathscr{H} f, \\
(f, \mathscr{H} g)=-(\mathscr{H} f, g), \quad(\mathscr{H} f, f)=0, \\
(\mathscr{H} f, \mathscr{H} g)=(f, g), \quad\|\mathscr{H} f\|=\|f\|, \\
\forall f \in H^{1}(\mathbb{R}), \quad \mathscr{H} f_{x}=(\mathscr{H} f)_{x} .
\end{gathered}
$$

Before we prove the existence of a compact random absorbing set, we first give some estimates about the solution $\bar{u}$ of problem $(P)$.

Let us introduce the following space to study the solution of problem $(P)$ :

$$
\begin{aligned}
X_{\sigma}(T)=\{ & u \in C\left(0, T ; H^{\sigma}(\mathbb{R})\right) \cap L^{2}\left(\mathbb{R} ; L^{\infty}([0, T])\right), \\
& D^{\sigma} \partial_{x} u \in L^{\infty}\left(\mathbb{R} ; L^{2}([0, T])\right), \\
& \left.\partial_{x} u \in L^{4}\left([0, T] ; L^{\infty}(\mathbb{R})\right)\right\},
\end{aligned}
$$

for some $\sigma<1$.

Lemma 4 (see [11]). Assume that $\Phi \in L_{2}^{0, \bar{\sigma}}$ for some $\bar{\sigma}>3 / 4$; then $\bar{u}$ is almost surely in $X_{\sigma}(T)$ for any $T>0$ and any $\sigma$ such that $3 / 4<\sigma<\bar{\sigma}$. More precisely,

$$
\begin{gathered}
\mathbb{E}\left(\sup _{t \in[0, T]}\|\bar{u}\|_{H_{x}^{\sigma}}^{2}\right) \leqslant C\|\Phi\|_{L_{2}^{0, \sigma}}^{2}, \quad \text { for any } \frac{3}{4}<\sigma \leqslant \bar{\sigma}, \\
\mathbb{E}\left(\left(\int_{0}^{T} \sup _{x \in \mathbb{R}}\left|\partial_{x} \bar{u}\right|^{4} d t\right)^{1 / 2}\right) \leqslant\|\Phi\|_{L_{2}^{0, \bar{\sigma}}}^{2}, \\
\mathbb{E}\left(\int_{\mathbb{R}} \sup _{t \in[0, T]}|\bar{u}|^{2} d x\right) \leqslant C(\bar{\sigma})\|\Phi\|_{L_{2}^{0, \bar{\sigma}} ;}^{2}
\end{gathered}
$$


let $0<\varepsilon<\inf \{\bar{\sigma}, 2\}$, and then

$$
\mathbb{E}\left(\sup _{x \in \mathbb{R}} \int_{0}^{T}\left|D^{\bar{\sigma}-\varepsilon} \partial_{x} \bar{u}\right|^{2} d t\right) \leqslant C(\varepsilon)\|\Phi\|_{L_{2}^{0, \bar{\sigma}}}^{2}
$$

one also has

$$
\mathbb{E}\left(\left(\int_{0}^{T} \sup _{x \in \mathbb{R}}|\bar{u}|^{4} d t\right)^{1 / 2}\right) \leqslant\|\Phi\|_{L_{2}^{0, \bar{\sigma}}}^{2},
$$

where $C(\varepsilon)$ and $C(\bar{\sigma})$ depend on $\varepsilon, \bar{\sigma}$, respectively.

Remark 5. We have to impose a stronger condition on $\Phi \in$ $L_{2}^{0,2}$ in the present paper than that on $\Phi \in L_{2}^{0,1}$ in [11]. Thus, the solution $\bar{u}$ of the linear problem is more regular, which will be used in proving the boundedness of $u$ in $H^{1}(\mathbb{R})$. More precisely, we can get

$$
\begin{aligned}
& \mathbb{E}\left(\left(\int_{0}^{T} \sup _{x \in \mathbb{R}}\left|\partial_{x}^{2} \bar{u}\right|^{4} d t\right)^{1 / 2}\right) \leqslant\|\Phi\|_{L_{2}^{0, \bar{\sigma}}}^{2}, \\
& \mathbb{E}\left(\sup _{x \in \mathbb{R}} \int_{0}^{T}\left|D \partial_{x} \bar{u}\right|^{2} d t\right) \leqslant C\|\Phi\|_{L_{2}^{0, \bar{\sigma}}}^{2} .
\end{aligned}
$$

4.1. Absorption in $L^{2}(\mathbb{R})$ at Time $t=-1$. Let $s<-1$ and $u_{s} \in L^{2}(\mathbb{R})$ be given, and let $v$ be the solution of (14) with initial condition $v(s, x)=v_{s}$.

Multiplying $(14)$ in $L^{2}(\mathbb{R})$ by $v$, we obtain

$$
\frac{1}{2} \frac{d}{d t}\|v\|^{2}+\lambda\|v\|^{2}=\int_{\mathbb{R}}\left(f v-\frac{1}{2} \bar{u}_{x} v^{2}-\overline{u u}_{x} v\right) d x
$$

It follows that

$$
\begin{aligned}
\frac{d}{d t}\|v\|^{2}+\lambda\|v\|^{2} \leqslant & \left\|\bar{u}_{x}\right\|_{L_{x}^{\infty}}\|v\|^{2} \\
& +\frac{2}{\lambda}\left\|\bar{u}_{x}\right\|_{L_{x}^{\infty}}^{2}\|\bar{u}\|^{2}+\frac{2}{\lambda}\|f\|^{2} .
\end{aligned}
$$

Using Gronwall Lemma for $s \leqslant-1$ and noticing Lemma 4, we get

$$
\begin{aligned}
& \|v(-1)\|^{2} \\
& \qquad\|v(s)\|^{2} \exp \left\{-\int_{s}^{-1}\left(\lambda-\left\|\bar{u}_{x}\right\|_{L_{x}^{\infty}}\right) d \tau\right\} \\
& +\int_{s}^{-1}\left(\frac{2}{\lambda}\left\|\bar{u}_{x}\right\|_{L_{x}^{\infty}}^{2}\|\bar{u}\|^{2}+\frac{2}{\lambda}\|f\|^{2}\right) \\
& \quad \times \exp \left\{-\int_{t}^{-1}\left(\lambda-\left\|u_{x}\right\|_{L_{x}^{\infty}}\right) d \tau\right\} d t
\end{aligned}
$$

$$
\begin{aligned}
\leqslant & \|v(s)\|^{2} \\
& \times \exp \left\{-(-1-s)^{3 / 4}\left(\lambda(-1-s)^{1 / 4}-\left\|\bar{u}_{x}\right\|_{L_{t}^{4}\left(L_{x}^{\infty}\right)}\right)\right\} \\
& +\frac{2}{\lambda} \int_{s}^{-1}\left\|\bar{u}_{x}\right\|_{L_{x}^{\infty}}^{2}\|\bar{u}\|^{2} \exp \left\{-\int_{t}^{-1}\left(\lambda-\left\|\bar{u}_{x}\right\|_{L_{x}^{\infty}}\right) d \tau\right\} d t \\
& +\frac{2}{\lambda} \int_{s}^{-1}\|f\|^{2} \exp \left\{-\int_{t}^{-1}\left(\lambda-\left\|\bar{u}_{x}\right\|_{L_{x}^{\infty}}\right) d \tau\right\} d t \\
\leqslant & \|v(s)\|^{2} e^{-(-1-s)^{3 / 4}\left(\lambda(-1-s)^{1 / 4}-\|\Phi\|_{\left.L_{2}^{0, \bar{\sigma}}\right)}\right.}+K_{1} .
\end{aligned}
$$

Noticing

$$
\begin{aligned}
& \int_{s}^{-1}\left\|\bar{u}_{x}\right\|_{L_{x}^{\infty}}^{2}\|\bar{u}\|^{2} \exp \left\{-\int_{t}^{-1}\left(\lambda-\left\|\bar{u}_{x}\right\|_{L_{x}^{\infty}}\right) d \tau\right\} d t \\
& \leqslant \sup _{t \in(-1, s)}\|\bar{u}\| \\
& \quad \times \int_{s}^{-1}\left\|\bar{u}_{x}\right\|_{L_{x}^{\infty}}^{2} \exp \left\{-\int_{t}^{-1}\left(\lambda-\left\|\bar{u}_{x}\right\|_{L_{x}^{\infty}}\right) d \tau\right\} d t \\
& \leqslant\|\Phi\|_{L_{2}^{0, \sigma}}^{2}\left(\|\Phi\|_{L_{2}^{0, \bar{\sigma}}}^{2}+C\right), \\
& \int_{s}^{-1}\|f\|^{2} \exp \left\{-\int_{t}^{-1}\left(\lambda-\left\|\bar{u}_{x}\right\|_{L_{x}^{\infty}}\right) d \tau\right\} d t \leqslant C\|f\|^{2},
\end{aligned}
$$

we get that $K_{1}=C\left(\|\Phi\|_{L_{2}^{0, \sigma}}^{2}\|\Phi\|_{L_{2}^{0, \bar{\sigma}}}^{2}+\|\Phi\|_{L_{2}^{0, \sigma}}^{2}+(2 / \lambda)\|f\|_{L_{x}^{2}}^{2}\right)$.

Then we have the following proposition.

Proposition 6. There exists a random radius $r_{1}(\omega)>0$, such that for all $\rho>0$ there exists $t(\omega) \leqslant-1$ such that the following holds $P$-a.s. For all $s \leqslant \bar{t}(\omega)$ and all $u_{s} \in L^{2}(\mathbb{R})$ with $\left\|u_{s}\right\| \leqslant \rho$, the solution $u$ of problem (5) with initial condition $u(s, x)=u_{s}$ satisfies the inequality

$$
\|u(-1, \omega)\|^{2} \leqslant r_{1}^{2}(\omega) .
$$

Proof. Given $\rho>0$, there exists $\bar{t}(\omega)$ such that

$$
e^{-(-1-s)^{3 / 4}\left(\lambda(-1-s)^{1 / 4}-\|\Phi\|_{L_{2}^{0, \bar{\sigma}}}^{2}\right)} \rho^{2} \leqslant 1
$$

for all $s \leqslant \bar{t}(\omega)$. Put

$$
r_{1}^{2}(\omega)=1+K_{1}+\|\bar{u}(-1)\|^{2}
$$

Then the proof of the proposition is completed.

We can also get an auxiliary estimate from (30) by the Gronwall Lemma with $s \leqslant t \leqslant 0$.

Consider the following:

$$
\begin{aligned}
&\|v(t)\|^{2} \leqslant\|v(s)\|^{2} \exp \left\{-\int_{s}^{t}\left(\lambda-\left\|\bar{u}_{x}\right\|_{L_{x}^{\infty}(\mathbb{R})}\right) d \tau\right\} \\
&+\frac{2}{\lambda} \int_{s}^{t}\left(\left\|\bar{u}_{x}\right\|_{L_{x}^{\infty}}^{2}\|\bar{u}\|^{2}+\|f\|^{2}\right) \\
& \times \exp \left\{-\int_{t}^{-1}\left(\lambda-\left\|\bar{u}_{x}\right\|_{L_{x}^{\infty}}\right) d \tau\right\} d t .
\end{aligned}
$$

This inequality will be useful in the following proof. 
4.2. Absorption in $H^{1}(\mathbb{R})$ at Time $t=0$. To obtain the $H^{1}$ estimate, we multiply (14) by $v_{x x}$ and integrate by part to get

$$
\begin{aligned}
\frac{1}{2} \frac{d}{d t} \| & v_{x}\left\|^{2}-\frac{1}{2}\left(\left(v^{2}\right)_{x}, v_{x x}\right)+\lambda\right\| v_{x} \|^{2} \\
& +\frac{3}{2}\left(\bar{u}_{x}, v_{x}^{2}\right)+\left(\bar{u}_{x}, v_{x}\right) \\
& +\left(\overline{u u}_{x x}, v_{x}\right)+\left(v \bar{u}_{x x}, v_{x}\right)-\left(f_{x}, v_{x}\right)=0 .
\end{aligned}
$$

(14) $\times v^{2} d x$ implies

$$
\begin{gathered}
\frac{1}{3} \frac{d}{d t} \int_{\mathbb{R}} v^{3} d x-\alpha \int_{\mathbb{R}}\left(v^{2}\right)_{x} \mathscr{H} v_{x} d x \\
\quad-\beta \int_{\mathbb{R}}\left(v^{2}\right)_{x} v_{x x} d x+\lambda \int_{x} v^{3} d x \\
+\left(\overline{u u}_{x}+v \bar{u}_{x}+\bar{u} v_{x}-f, v^{2}\right)=0 .
\end{gathered}
$$

\section{Moreover}

$$
\begin{gathered}
\frac{d}{d t} \int_{\mathbb{R}} v \mathscr{H} v_{x} d x+\int_{\mathbb{R}}\left(v^{2}\right)_{x} \mathscr{H} v_{x} d x+2 \int_{\mathbb{R}} \bar{u} v_{x} \mathscr{H} v_{x} d x \\
-2\left(f-\lambda v-\overline{u u}_{x}, \mathscr{H} v_{x}\right)=0 .
\end{gathered}
$$

Combining (37), (38), and (39) $(2 \beta(37)+(38)+\alpha(39))$, we have

$$
\begin{aligned}
\frac{d}{d t} \int_{\mathbb{R}} & \left(\beta\left|v_{x}\right|^{2}+\frac{1}{3} v^{3}+\alpha v \mathscr{H} v_{x}\right) d x+2 \beta \lambda\left\|v_{x}\right\|^{2} \\
& +\lambda \int_{\mathbb{R}} v^{3} d x+2 \alpha \lambda \int_{\mathbb{R}} v \mathscr{H} v_{x} d x \\
& +3 \beta\left(\bar{u}_{x}, v_{x}^{2}\right)+2 \alpha\left(\bar{u}, v_{x} \mathscr{H} v_{x}\right) \\
& +2 \beta\left(\bar{u}_{x}^{2}+\overline{u u}_{x x}+v \bar{u}_{x x}-f_{x}, v_{x}\right) \\
& +\left(\overline{u u}_{x}+v \bar{u}_{x}+\bar{u} v_{x}-f, v^{2}\right) \\
& -2 \alpha\left(f-\overline{u u}_{x}, \mathscr{H} v_{x}\right)=0 .
\end{aligned}
$$

Denote

$$
\varphi(v)=\int_{\mathbb{R}}\left(\beta\left|v_{x}\right|^{2}+\frac{1}{3} v^{3}+\alpha v \mathscr{H} v_{x}\right) d x .
$$

And noticing that

$$
\begin{gathered}
-\left(v_{x} \bar{u}, v^{2}\right)=2\left(v_{x} \bar{u}, v^{2}\right)+\left(v \bar{u}_{x}, v^{2}\right), \\
-\left(v_{x} \bar{u}, v^{2}\right)=\frac{1}{3}\left(v \bar{u}_{x}, v^{2}\right), \\
\left|3\left(\bar{u}_{x}, v_{x}^{2}\right)\right| \leqslant 3\left\|\bar{u}_{x}\right\|_{L_{x}^{\infty}}\left\|v_{x}\right\|_{L_{x}^{2}}^{2},
\end{gathered}
$$$$
\left|2 \alpha\left(\bar{u}, v_{x} \mathscr{H} v_{x}\right)\right| \leqslant 2|\alpha|\|\bar{u}\|_{L^{\infty}}\left\|v_{x}\right\|^{2} \text {, }
$$

we deduce that

$$
\begin{aligned}
\frac{d}{d t} & \varphi(v)+\left(\lambda-\frac{2|\alpha|}{\beta}\|\bar{u}\|_{L_{x}^{\infty}}-3\left\|\bar{u}_{x}\right\|_{L_{x}^{\infty}}\right) \varphi(v)+\beta \lambda\left\|v_{x}\right\|^{2} \\
\leqslant & -\left(\frac{2}{3} \lambda+\frac{2|\alpha|}{3 \beta}\|\bar{u}\|_{L_{x}^{\infty}}+\left\|\bar{u}_{x}\right\|_{L_{x}^{\infty}}\right) \int_{\mathbb{R}} v^{3} d x \\
& -\alpha\left(\lambda+\frac{2|\alpha|}{\beta}\|\bar{u}\|_{L_{x}^{\infty}}+3\left\|\bar{u}_{x}\right\|_{L_{x}^{\infty}}\right) \\
& \times \int_{\mathbb{R}} v \mathscr{H} v_{x} d x-2 \beta\left(\bar{u}_{x}^{2}+\overline{u u}_{x x}+v \bar{u}_{x x}-f_{x}, v_{x}\right) \\
& -\left(\overline{u u}_{x}+\frac{2}{3} v \bar{u}_{x}-f, v^{2}\right) \\
& +2 \alpha\left(f-\overline{u u}_{x}, \mathscr{H} v_{x}\right) .
\end{aligned}
$$

Now we stop to estimate the right hand side of (43) term by term:

$$
\begin{aligned}
& \left|2 \beta\left(v \bar{u}_{x x}, v_{x}\right)\right| \leqslant C\left\|\bar{u}_{x x}\right\|_{L_{x}^{\infty}}^{2}\|v\|_{L_{x}^{2}}^{2}+\frac{\beta \lambda}{12}\left\|v_{x}\right\|_{L_{x}^{2}}^{2}, \\
& \left|2 \beta\left(\bar{u}_{x}^{2}, v_{x}\right)\right| \leqslant 2\left\|\bar{u}_{x}\right\|_{L_{x}^{\infty}}\left\|\bar{u}_{x}\right\|_{L_{x}^{2}}\left\|v_{x}\right\|_{L_{x}^{2}} \\
& \leqslant C\left\|\bar{u}_{x}\right\|_{L_{x}^{\infty}}^{2}\left\|\bar{u}_{x}\right\|_{L_{x}^{2}}^{2}+\frac{\beta \lambda}{12}\left\|v_{x}\right\|_{L_{x}^{2}}^{2}, \\
& \left|2 \beta\left(\overline{u u}_{x x}, v_{x}\right)\right| \leqslant C\left\|\overline{u u}_{x x}\right\|_{L_{x}^{2}}^{2}+\frac{\beta \lambda}{12}\left\|v_{x}\right\|_{L_{x}^{2}}^{2}, \\
& \left|-\frac{2}{3}\left(v \bar{u}_{x}, v^{2}\right)\right|+\left\|\bar{u}_{x}\right\|_{L_{x}^{\infty}}\|v\|_{L^{3}(\mathbb{R})}^{3} \\
& \leqslant\left|\bar{u}_{x}\right|_{L_{x}^{\infty}}|v|_{L_{x}^{2}}^{5 / 2}\left|v_{x}\right|_{L_{x}^{2}}^{1 / 2} \\
& \leqslant C\left\|\bar{u}_{x}\right\|_{L_{x}^{\infty}}^{4}+|v|_{L_{x}^{2}}^{5}+\frac{\beta \lambda}{12}\left|v_{x}\right|_{L_{x}^{2}}^{2}, \\
& \left|-\left(\bar{u}_{x}, v^{2}\right)\right| \leqslant\left\|\bar{u}_{x}\right\|_{L_{x}^{\infty}}\|\bar{u}\|_{L_{x}^{2}}\|v\|_{L_{x}^{4}}^{2} \\
& \leqslant\left\|\bar{u}_{x}\right\|_{L_{x}^{\infty}}\|\bar{u}\|_{L_{x}^{2}}\|v\|_{L_{x}^{2}}^{3 / 2}\left\|v_{x}\right\|_{L_{x}^{2}}^{1 / 2} \\
& \leqslant\left\|\bar{u}_{x}\right\|_{L_{x}^{\infty}}^{4 / 3}\|\bar{u}\|_{L_{x}^{2}}^{4 / 3}\|v\|_{L_{x}^{2}}^{2}+\frac{\beta \lambda}{12}\left\|v_{x}\right\|_{L_{x}^{2}}^{2} \\
& \leqslant C(\lambda)\left\|\bar{u}_{x}\right\|_{L_{x}^{\infty}}^{4}+\|\bar{u}\|_{L_{x}^{2}}^{2}\|v\|_{L_{x}^{2}}^{3}+\frac{\beta \lambda}{12}\left\|v_{x}\right\|_{L_{x}^{2}}^{2}, \\
& \left|-2 \beta\left(f_{x}, v_{x}\right)\right| \leqslant C(\lambda)\left\|f_{x}\right\|_{L_{x}^{2}}^{2}+\frac{\beta \lambda}{12}\left\|v_{x}\right\|_{L_{x}^{2}}^{2}, \\
& \left|\left(f, v^{2}\right)\right| \leqslant C(\lambda)\|f\|_{L_{x}^{2}}^{4 / 3}\|v\|_{L_{x}^{2}}^{2}+\frac{\beta \lambda}{12}\left\|v_{x}\right\|_{L_{x}^{2}}^{2}, \\
& \frac{2}{3} \lambda\|v\|_{L^{3}(\mathbb{R})}^{3} \leqslant C(\lambda)\|v\|_{L_{x}^{2}}^{10 / 3}+\frac{\beta \lambda}{12}\left\|v_{x}\right\|_{L_{x}^{2}}^{2}, \\
& \frac{2|\alpha|}{3 \beta}\|\bar{u}\|_{\infty} \int_{\mathbb{R}}|v|^{3} d x \leqslant C\left\|\bar{u}_{x}\right\|^{2}+\|\bar{u}\|^{2}
\end{aligned}
$$




$$
\begin{gathered}
+\|v\|^{20 / 3}+\frac{\beta \lambda}{12}\left\|v_{x}\right\|_{L_{x}^{2}}^{2}, \\
\left|\alpha \lambda \int_{\mathbb{R}}\left(v \mathscr{H} v_{x}+2 f \mathscr{H} v_{x}\right) d x\right| \\
\leqslant C\left(\|v\|^{2}+\|f\|^{2}\right)+\frac{\beta \lambda}{12}\left\|v_{x}\right\|_{L_{x}^{2}}^{2}, \\
\left|\frac{2 \alpha^{2}}{\beta}\|\bar{u}\|_{\infty} \int_{\mathbb{R}} v \mathscr{H} v_{x} d x\right| \\
\leqslant C\left\|\bar{u}_{x}\right\|^{2}+\|\bar{u}\|^{2}\|v\|^{4}+\frac{\beta \lambda}{12}\left\|v_{x}\right\|_{L_{x}^{2}}^{2}, \\
\left|\left(\overline{u u}_{x}, \mathscr{H} v_{x}\right)\right|=\left|-\left(\bar{u}_{x}^{2}, \mathscr{H} v\right)-\left(\bar{u} \bar{u}_{x x}, \mathscr{H} v\right)\right| \\
\leqslant C\left(\|v\|^{2}+\left\|\bar{u} x_{x x}\right\|^{2}+\left\|\bar{u}_{x}\right\|^{4}\right)+\frac{\beta \lambda}{12}\left\|v_{x}\right\|_{L_{x}^{2}}^{2} . \\
\text { Then combining the above estimates, we get } \\
\frac{d}{d t} \varphi(v)+\left(\lambda-\frac{2|\alpha|}{\beta}\|\bar{u}\|_{L_{x}^{\infty}}-3\left\|\bar{u}_{x}\right\|_{L_{x}^{\infty}}\right) \varphi(v) \\
\leqslant C\left(\left\|\bar{u}_{x x}\right\|_{L_{x}^{\infty}}^{2}\|v\|_{L_{x}^{2}}^{2}+\left\|\bar{u}_{x}\right\|_{L_{x}^{\infty}}^{2}\left\|\bar{u}_{x}\right\|_{L_{x}^{2}}^{2}\right. \\
+\left\|\bar{u} \bar{u}_{x x}\right\|_{L_{x}^{2}}^{2}+\left\|\bar{u}_{x}\right\|_{L_{x}^{2}}^{2}+\|v\|_{L_{x}^{2}}^{5} \\
+\left\|\bar{u}_{x}\right\|_{L_{x}^{\infty}}^{4}+\|\bar{u}\|_{L_{x}^{2}}^{2}\|v\|_{L_{x}^{2}}^{3} \\
+\|f\|_{L_{x}^{2}}^{4 / 3}\|v\|_{L_{x}^{2}}^{2}+\|v\|_{L_{x}^{2}}^{10 / 3}+\|\bar{u}\|^{2} \\
\left.+\|v\|^{20 / 3}+\|\bar{u}\|^{2}\|v\|^{4}\right) .
\end{gathered}
$$

Applying the Gronwall Lemma for $s \leqslant 0$, we find

$$
\begin{aligned}
& \varphi(v(0)) \leqslant \varphi(v(s)) e^{-\int_{s}^{0}\left(\lambda-(2|\alpha| / \beta)\|\bar{u}\|_{L_{x}^{\infty}}^{\infty}-3\left\|\bar{u}_{x}\right\|_{L_{x}^{\infty}}\right) d \tau} \\
& +C \int_{s}^{0}\left(\left\|\bar{u}_{x x}\right\|_{L_{x}^{\infty}}^{2}\|v\|_{L_{x}^{2}}^{2}+\left\|\bar{u}_{x}\right\|_{L_{x}^{\infty}}^{2}\left\|\bar{u}_{x}\right\|_{L_{x}^{2}}^{2}\right. \\
& +\|\bar{u}\|_{L_{x}^{2}}^{2}+\left\|\bar{u}_{x x}\right\|_{L_{x}^{2}}^{2}+\left\|\bar{u}_{x}\right\|_{L_{x}^{\infty}}^{4}+\|v\|_{L_{x}^{2}}^{5} \\
& +\left\|\bar{u}_{x}\right\|_{L_{x}^{\infty}}^{4}+\|\bar{u}\|_{L_{x}^{2}}^{2}\|v\|_{L_{x}^{2}}^{3}+\left\|f_{x}\right\|_{L_{x}^{2}}^{2} \\
& \left.+\|f\|_{L_{x}^{2}}^{4 / 3}\|v\|_{L_{x}^{2}}^{2}+\|v\|_{L_{x}^{2}}^{10 / 3}\right) \\
& \times e^{-\int_{t}^{0}\left(\lambda-(2|\alpha| / \beta)\|\bar{u}\|_{L_{x}^{\infty}}^{\infty}-3\left\|\bar{u}_{x}\right\|_{L_{x}^{\infty}}\right) d \tau} d t .
\end{aligned}
$$

We can read the boundedness of the right hand side of (46) from the following estimates. Using (36), we have

$$
\begin{aligned}
& \int_{s}^{0}\left\|\bar{u}_{x x}\right\|_{L_{x}^{\infty}}^{2}\|v(t)\|_{L_{x}^{2}}^{2} e^{-\int_{t}^{0}\left(\lambda-(2|\alpha| / \beta)\|\bar{u}\|_{L_{x}^{\infty}}-3\left\|\bar{u}_{x}\right\|_{L_{x}^{\infty}}\right) d \tau} d t \\
& \quad \leqslant \int_{s}^{0}\left\|\bar{u}_{x x}\right\|_{L_{x}^{\infty}}^{2}
\end{aligned}
$$

$$
\begin{aligned}
& \quad \times\left\{\|v(s)\|^{2} \exp \left(-\int_{s}^{t}\left(\lambda-\left\|u_{x}\right\|_{L_{x}^{\infty}(\mathbb{R})}\right) d \tau\right)+C\right\} \\
& \times e^{-\int_{t}^{0}\left(\lambda-(2|\alpha| / \beta)\|\bar{u}\|_{L_{x}^{\infty}}-3\left\|\bar{u}_{x}\right\|_{L_{x}^{\infty}}\right) d \tau} d t \\
& \leqslant \int_{s}^{0}\left\|\bar{u}_{x x}\right\|_{L_{x}^{\infty}}^{2}\|v(s)\|^{2} e^{-(-s)^{3 / 4}\left((-s)^{1 / 4} \lambda-4\left\|\bar{u}_{x}\right\|_{L_{L}^{4}\left(L_{x}^{\infty}\right)}\right)} d t \\
& +C \int_{s}^{0}\left\|\bar{u}_{x x}\right\|_{L_{x}^{\infty}}^{2} e^{-\int_{t}^{0}\left(\lambda-(2|\alpha| / \beta) \| \bar{u}_{\left.L_{x}^{\infty}-3\left\|\bar{u}_{x}\right\|_{L_{x}^{\infty}}\right)} d \tau\right.} d t \\
& \leqslant C\left\|\bar{u}_{x x}\right\|_{L_{t}^{4}\left(L_{x}^{\infty}\right)}^{4} .
\end{aligned}
$$

We also have

$$
\begin{aligned}
& \int_{s}^{0} \int_{-\infty}^{+\infty}|\bar{u}|^{2}\left|\bar{u}_{x x}\right|^{2} d x d t \\
& \quad \leqslant \int_{-\infty}^{+\infty} \text { ess. } \sup _{t \in[s, 0]}|\bar{u}|^{2} \int_{s}^{0}\left|\bar{u}_{x x}\right|^{2} d x d t \\
& \quad \leqslant \int_{-\infty}^{+\infty} \text { ess. } \sup _{t \in[s, 0]}|\bar{u}|^{2} d x \text { ess.sup } \int_{x \in \mathbb{R}} \int_{s}^{0}\left|\bar{u}_{x x}\right|^{2} d t . \\
& \quad=\|\bar{u}\|_{W_{x}^{2, \infty}\left(L_{t}^{2}\right)}^{2}\|\bar{u}\|_{L_{x}^{2}\left(L_{t}^{2 \infty}\right)}^{2} .
\end{aligned}
$$

The boundedness of other terms about the right hand side of (46) is obvious. Noticing (25)-(28), we get

$$
\begin{aligned}
\varphi(v(0)) & \leqslant \varphi(v(s)) e^{-\int_{s}^{0}\left(\lambda-3\left\|\bar{u}_{x}\right\|_{L_{x}^{\infty}}\right) d \tau}+C K_{2} \\
& \leqslant \varphi(v(s)) e^{-\int_{s}^{0}\left(\lambda-3\left\|\bar{u}_{x}\right\|_{L_{x}^{\infty}}\right) d \tau}+C K_{3},
\end{aligned}
$$

where

$$
\begin{gathered}
K_{2}=C\left(\left\|\bar{u}_{x x}\right\|_{W_{x}^{2, \infty}\left(L_{t}^{2}\right)}^{2}\|\bar{u}\|_{L_{x}^{2}\left(L_{t}^{\infty}\right)}^{2}\right. \\
+\left\|\bar{u}_{x x}\right\|_{L_{t}^{4}\left(L_{x}^{\infty}\right)}^{4}+\|u\|_{L_{t}^{\infty}\left(L_{x}^{2}\right)}^{2}\|\bar{u}\|_{L_{t}^{4}\left(L_{x}^{\infty}\right)}^{4}\left(\|\bar{u}\|_{L_{x}^{2}\left(L_{t}^{\infty}\right)}^{2}+\left\|\bar{u}_{x}\right\|_{L_{t}^{4}\left(L_{x}^{\infty}\right)}^{4}\right. \\
\left.+\|\bar{u}\|_{L_{x}^{2}\left(L_{x}^{\infty}\right)}^{2}+\left\|f_{x}\right\|_{L_{x}^{2}}^{2}\right) \\
K_{3}=\|\Phi\|_{L_{2}^{0, \bar{\sigma}}}^{4}+\|\Phi\|_{L_{2}^{0, \bar{\sigma}}}^{6}+\left\|f_{x}\right\|_{L_{x}^{2}}^{2} .
\end{gathered}
$$

Then we get the following proposition.

Proposition 7. There exists a random variable $r_{2}(\omega)>0$, such that for all $\rho>0$ there exists $\widetilde{t}(\omega) \leqslant 0$ such that the following holds $P$-a.s. For all $s \leqslant \widetilde{t}(\omega)$ and all $u_{s} \in H^{1}(\mathbb{R})$ with $\left\|u_{s}\right\|_{H^{1}(\mathbb{R})} \leqslant \rho$, $\varphi$ satisfies

$$
\varphi(v(0)) \leqslant r_{2}(\omega) .
$$

Proof. Given $\rho>0$, there exists $\widetilde{t}(\omega)$ such that

$$
\varphi(v(s)) e^{-\int_{s}^{0}\left(\lambda-(2|\alpha| / \beta)\|\bar{u}\|_{L_{x}^{\infty}}-3\left\|\bar{u}_{x}\right\|_{L_{x}^{\infty}}\right) d \tau} \leqslant 1,
$$


for all $s \leqslant \widetilde{t}(\omega)$. Put

$$
r_{2}(\omega)=1+K_{3} .
$$

Then the proof of the proposition is completed.

Corollary 8. There exists a random variable $r_{3}(\omega)>0$, such that the solution $u\left(t, \omega ; s, u_{s}\right)$ of problem (5) with the initial condition $u(s, x)=u_{s}$ satisfies

$$
\left\|u\left(0, \omega ; s, u_{s}\right)\right\|_{H^{1}(\mathbb{R})} \leqslant r_{3}(\omega) .
$$

Proof. We can deduce from the presentation of $\varphi(v)$ that

$$
\frac{2}{3}\left\|v_{x}\right\|^{2}-\frac{1}{2^{8 / 3}}\|v\|^{10 / 3} \leqslant \varphi(v) .
$$

Then

$$
\left\|v_{x}(0)\right\|^{2} \leqslant \frac{3}{2}+K_{1}+\frac{3}{2^{10 / 3}}\|v(-1)\|^{10 / 3} .
$$

By $v(t)=u(t)-\bar{u}(t)$,

$$
\begin{aligned}
\left\|u_{x}(0)\right\|^{2} & \leqslant 2\left(\left\|v_{x}(0)\right\|^{2}+\left\|\bar{u}_{x}(0)\right\|^{2}\right) \\
& \leqslant 2\left(\frac{3}{2}+K_{1}+\frac{3}{2^{10 / 3}}\|v(-1)\|^{10 / 3}+\left\|\bar{u}_{x}(0)\right\|^{2}\right) \\
& =r_{3}(\omega) .
\end{aligned}
$$

Then the proof of the corollary is completed.

4.3. Construct the Attractor. Before we construct the random attractor, it is worthwhile to point out that the domain about the space variable is unbounded which makes the embedding $H^{1}(\mathbb{R}) \rightarrow L^{2}(\mathbb{R})$ to be noncompact. Fortunately, we can get weak compactness in the space $H^{1}(\mathbb{R})$ by Lemma 9 given below. Motivated by the definition of weak attractor for the deterministic system in [12], we extend this definition to the RDS. Then we get weak random global attractor for (5) with initial condition $u(s, x)=u_{s}$.

Lemma 9 (see [13]). The solution operator $\{S(t)\}_{t \in \mathbb{R}}$ is weakly continuous in $H^{1}(\mathbb{R})$ in the sense that if $v_{0 n}$ converges weakly in $H^{1}(\mathbb{R})$ to some $v_{0}$ as $n \rightarrow \infty$, then $S(t) v_{0 n}$ converges to $S(t) v_{0}$ weakly in $H^{1}(\mathbb{R})$ for all $t \in \mathbb{R}$.

Having Proposition 7, Corollary 8, and Lemma 9 in hand, one begins to construct the weak random attractor in space $H^{1}(\mathbb{R})$.

From Section 4.2, one gets an RDS for the stochastic weakly damped, forced $\mathrm{KdV}-\mathrm{BO}$ equation with additive noise; that is,

$$
u\left(t, \omega ; s, u_{s}\right)=S(t, s ; \omega) u_{s}=v\left(t, \omega ; s ; v_{s}\right)+\bar{u}(t, \omega) .
$$

One recalls in [8] that if $B$ and $A$ are random sets such that for $P$-almost all $\omega$ there exists a time $T_{B}(\omega)$ such that for all $t \leqslant t_{B}(\omega)$

$$
\Psi\left(t, \theta_{-t} \omega\right) B\left(\theta_{-t} \omega\right) \subset A(\omega),
$$

then $A$ is said to absorb $B$. Of course, if $A$ absorbs $B$, then $A$ attracts $B$.

Denote

$$
A_{1}=\left\{u \in H^{1}(\mathbb{R}),\left\|u_{x}\right\|^{2} \leqslant r_{3}(\omega)\right\} .
$$

It is easy to see that $A_{1}$ is an absorbing set for $\operatorname{RDS}\{\Psi(t, \omega)\}$ in $H^{1}(\mathbb{R})$. Furthermore it is a weak compactness according to Lemma 9.

Proposition 10. Let

$$
\mathscr{A}(\omega)=\overline{\mathrm{U}_{B \subset X} \Lambda_{B}(\omega)}
$$

where $\Lambda_{B}(\omega):=\cap_{s \geqslant 0} \overline{U_{t \geqslant s} \phi\left(t, \theta_{-t} \omega\right) B}$ is the omega-limit set of $B$. Here the closures are taken with respect to the weak topology of $H^{1}(\mathbb{R})$. Then $\mathscr{A}(\omega)$ is included in $A_{1}$ and is nonempty. It is invariant by $\Psi(t, \omega)$; that is,

$$
\Psi(t, \omega) \mathscr{A}(\omega)=\mathscr{A}\left(\theta_{t} \omega\right), \quad \forall t>0 .
$$
facts.

The proof of Proposition 10 is easy using the following

(a) $\Psi(t, \omega)$ is weakly continuous on $H^{1}(\mathbb{R})$, which is an obvious corollary of Lemma 9.

(b) A point $b \in \mathscr{A}(\omega)$ if and only if there exist two sequences $\left(s_{n}\right)_{n \in \mathbb{N}} \in \mathbb{R},\left(x_{n}\right)_{n \in \mathbb{N}} \in \mathscr{A}(\omega)$ such that $s_{n} \rightarrow-\infty$ and $\phi\left(s_{n}\right) x_{n} \rightarrow b$.

Then $\mathscr{A}(\omega)$ has the following properties:

(i) $\mathscr{A}(\omega)$ is random compact set;

(ii) $\Psi(t, \omega) \mathscr{A}(\omega)=\mathscr{A}\left(\theta_{t} \omega\right)$ for all $t \geqslant 0$;

(iii) $\mathscr{A}(\omega)$ attracts all deterministic bounded sets under the sense that

$$
\lim _{t \rightarrow \infty} d^{w}\left(\Psi\left(t, \theta_{t} \omega\right) B, \mathscr{A}\right)=0,
$$

where $d^{w}$ denotes the distance in the weak topology of $H^{1}(\mathbb{R})$.

Then we get Theorem 1 in this paper.

\section{Conflict of Interests}

The author declares that there is no conflict of interests regarding the publication of this paper.

\section{Acknowledgment}

This work is supported by the National Natural Science Foundation of China (no. 11101309).

\section{References}

[1] B. Guo and Z. Huo, "The well-posedness of the Kortewegde Vries-Benjamin-Ono equation," Journal of Mathematical Analysis and Applications, vol. 295, no. 2, pp. 444-458, 2004.

[2] F. Linares, " $L^{2}$ global well-posedness of the initial value problem associated to the Benjamin equation," Journal of Differential Equations, vol. 152, no. 2, pp. 377-393, 1999. 
[3] R. L. Herman, “The stochastic, damped KdV equation," Journal of Physics A: Mathematical and General, vol. 23, no. 7, pp. 1063$1084,1990$.

[4] M. Scalerandi, A. Romano, and C. A. Condat, "Korteweg-de Vries solitons under additive stochastic perturbations," Physical Review E: Statistical Physics, Plasmas, Fluids, and Related Interdisciplinary Topics, vol. 58, no. 4, pp. 4166-4173, 1998.

[5] B. Guo and Z. Huo, "The global attractor of the damped, forced generalized Korteweg de Vries-Benjamin-Ono equation in $L^{2}$," Discrete and Continuous Dynamical Systems A, vol. 16, no. 1, pp. 121-136, 2006.

[6] G. Wang and B. Guo, "Well-posedness of stochastic Kortewegde Vries-Benjamin-Ono equation," Frontiers of Mathematics in China, vol. 5, no. 1, pp. 161-177, 2010.

[7] L. Arnold, Random Dynamical Systems, Springer, Berlin, Germany, 1998.

[8] H. Crauel and F. Flandoli, "Attractors for random dynamical systems," Probability Theory and Related Fields, vol. 100, no. 3, pp. 365-393, 1994.

[9] H. Crauel, A. Debussche, and F. Flandoli, "Random attractors," Journal of Dynamics and Differential Equations, vol. 9, no. 2, pp. 307-341, 1997.

[10] G. da Prato and J. Zabczyk, Stochastic Equations in Infinite Dimensions, Cambridge University Press, 1992.

[11] A. de Bouard and A. Debussche, "On the stochastic Kortewegde Vries equation," Journal of Functional Analysis, vol. 154, no. 1, pp. 215-251, 1998.

[12] J.-M. Ghidaglia, "Finite-dimensional behavior for weakly damped driven Schrödinger equations," Annales de l'Institut Henri Poincaré. Analyse Non Linéaire, vol. 5, no. 4, pp. 365-405, 1988.

[13] R. Rosa, "The global attractor of a weakly damped, forced Korteweg-de Vries equation in $H^{1}(\mathbb{R})$," Matemática Contemporânea, vol. 19, pp. 129-152, 2000. 


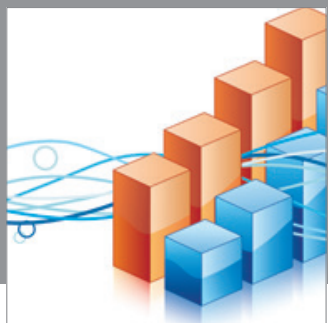

Advances in

Operations Research

mansans

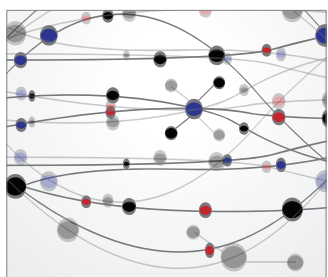

The Scientific World Journal
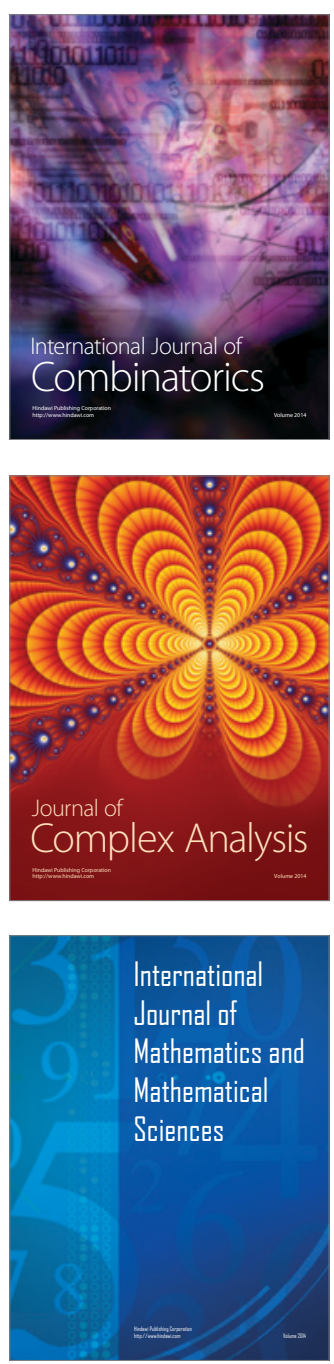
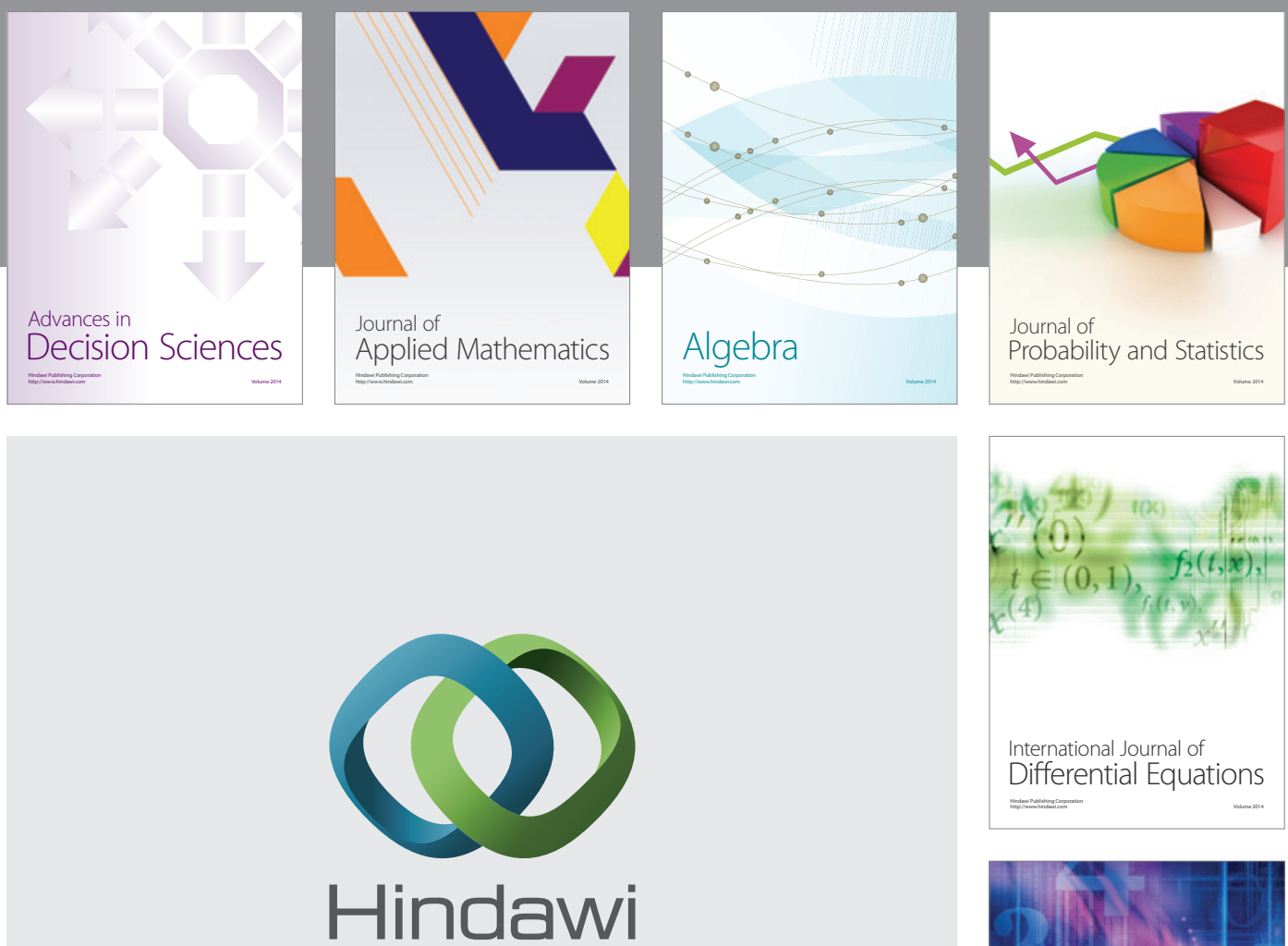

Submit your manuscripts at http://www.hindawi.com
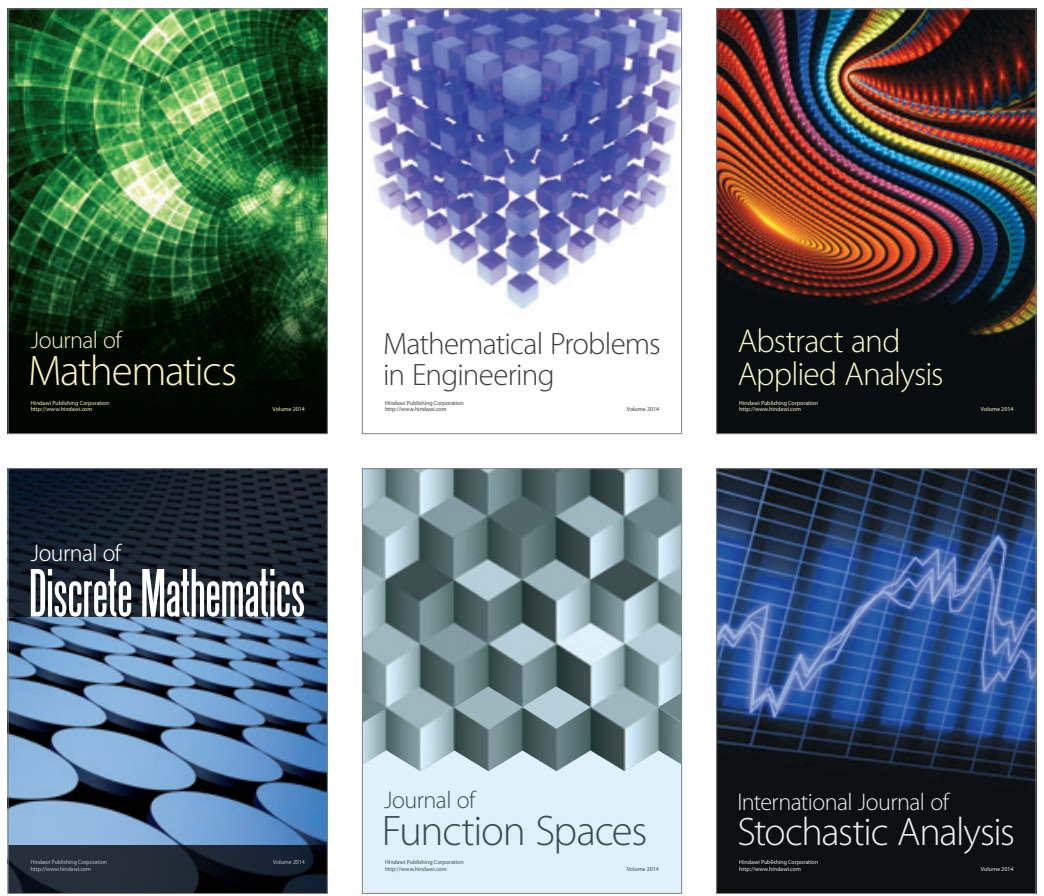

Journal of

Function Spaces

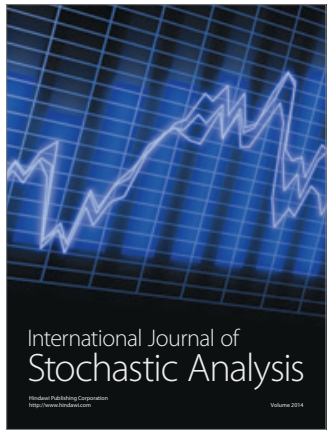

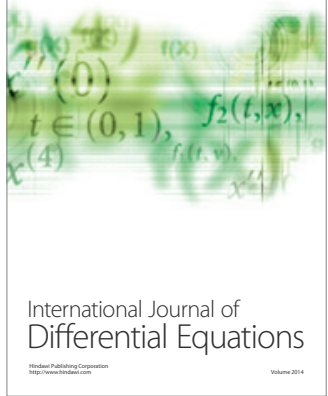
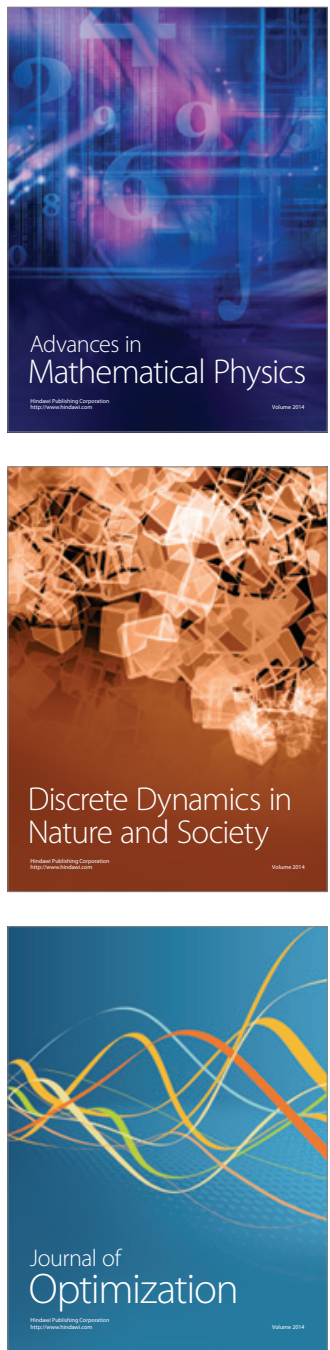